\title{
Synaptic plasticity controls the emergence of population-wide invariant representations in balanced network models
}

\author{
L. Bernáez Timón $\odot,,^{1,2, *}$ P. Ekelmans, ${ }^{4,2,1}$ S. Konrad $\odot,{ }^{2,5}$ A. Nold $\odot,{ }^{3,2}$ and T. Tchumatchenko $\oplus^{1,3,2, \dagger}$ \\ ${ }^{1}$ University Medical Center of the Johannes Gutenberg-University Mainz, Institute for Physiological Chemistry, \\ Duesbergweg 6, 55128 Mainz, Germany \\ ${ }^{2}$ Max Planck Institute for Brain Research, Theory of Neural Dynamics, Max-von-Laue-Straße 4, 60438 Frankfurt am Main, Germany \\ ${ }^{3}$ University of Bonn Medical Center, Institute of Experimental Epileptology and Cognition Research, Venusberg-Campus 1, \\ 53127 Bonn, Germany \\ ${ }^{4}$ Goethe University Frankfurt, Frankfurt Institute for Advanced Studies, Ruth-Moufang-Straße 1, 60438 Frankfurt am Main, Germany \\ ${ }^{5}$ Heidelberg University, Institute for Theoretical Physics, Philosophenweg 16, 69120 Heidelberg, Germany
}

(Received 25 June 2021; revised 24 November 2021; accepted 24 January 2022; published 28 February 2022)

\begin{abstract}
The intensity and the features of sensory stimuli are encoded in the activity of neurons in the cortex. In the visual and piriform cortices, the stimulus intensity rescales the activity of the population without changing its selectivity for the stimulus features. The cortical representation of the stimulus is therefore intensity invariant. This emergence of network-invariant representations appears robust to local changes in synaptic strength induced by synaptic plasticity, even though (i) synaptic plasticity can potentiate or depress connections between neurons in a feature-dependent manner, and (ii) in networks with balanced excitation and inhibition, synaptic plasticity determines the nonlinear network behavior. In this study we investigate the consistency of invariant representations with a variety of synaptic states in balanced networks. By using mean-field models and spiking network simulations, we show how the synaptic state controls the emergence of intensity-invariant or intensity-dependent selectivity. In particular, we demonstrate that an effective power-law synaptic transformation at the population level is necessary for invariance. In a range of firing rates, purely depressing short-term synapses fulfills this condition, and in this case, the network is contrast-invariant. Instead, facilitating short-term plasticity generally narrows the network selectivity. We found that facilitating and depressing short-term plasticity can be combined to approximate a power-law that leads to contrast invariance. These results explain how the physiology of individual synapses is linked to the emergence of invariant representations of sensory stimuli at the network level.
\end{abstract}

DOI: 10.1103/PhysRevResearch.4.013162

\section{INTRODUCTION}

Transformations performed by primary sensory cortices constitute one of the first computational steps towards the sensory perception of the environment [1-3]. One fundamental cortical computation is the representation of the stimulus intensity [4-7]. For example, an animal may encounter an object which elicits visual stimuli of different brightness, scents of different concentrations, or sound frequencies of different volumes. Despite these differences in stimulus intensity, many animals still reliably identify the object [8]. How does the cortex represent the stimulus intensity (its brightness, concentration, or volume) without compromising information about the stimulus identity (i.e., its orientation, scent, or frequency)? In the past decades, the representation of the stimulus intensity was extensively studied at the single-neuron level $[4,7,9,10]$.

\footnotetext{
*1bernaeztimon@gmail.com

${ }^{\dagger}$ tatjana.tchumatchenko@uni-mainz.de
}

Published by the American Physical Society under the terms of the Creative Commons Attribution 4.0 International license. Further distribution of this work must maintain attribution to the author(s) and the published article's title, journal citation, and DOI. Open access publication funded by the Max Planck Society.
Here we investigate how changes in the plasticity, i.e., the synaptic connections between neurons, influence these stimulus representations at the network level.

Primary cortices represent sensory stimuli through the combined activity of neurons which are selective towards features such as orientation [9], odor [11], or frequency [12]. These neurons are preferentially connected to neurons with similar preferred features [13] through plastic synapses $[14,15]$ and often respond nonlinearly to the stimulus intensity [16]. At the network level, the collective pattern of activity of these neurons can be described by the product of two functions [16,17]: the network selectivity, i.e., its dependence on the identity of the stimulus, and the network susceptibility, i.e., its dependence on the intensity of the stimulus. In the visual $[16,17]$ and piriform cortices [6] (but not in the auditory cortex $[18,19])$, the activity of the network at high stimulus intensities is a rescaled version of the low-intensity representation. This phenomenon is referred to as network contrast invariance in the visual cortex $[16,17]$ and concentration invariance in the piriform cortex [6]. Notably, contrast-invariant representations have also been observed and studied at the single-neuron level $[4,7,10,20]$.

However, network contrast invariance does not necessarily emerge from the single-neuron property of contrast invariance (Fig. 1) $[4,7,10,20]$ : counterintuitively, the summed activity of 


\section{(a) Recurrent networks of contrast-invariant neurons may not be invariant}

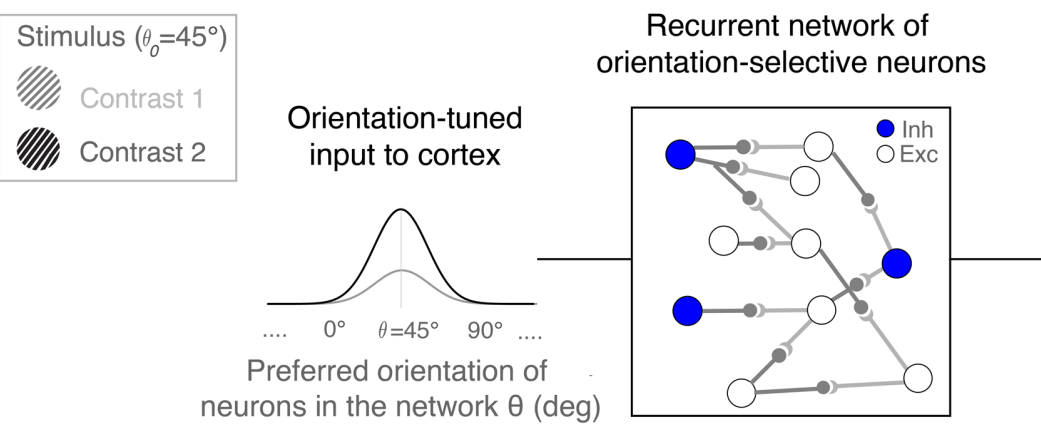

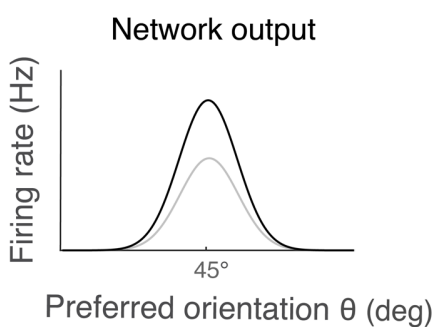

(b) Synaptic plasticity can control the network selectivity
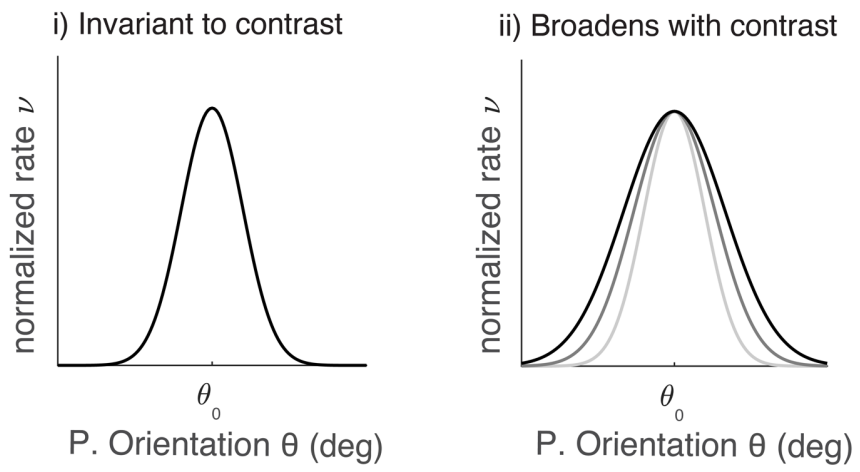

iii) Narrows with contrast

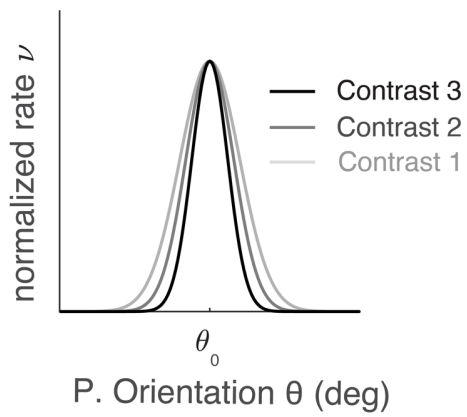

FIG. 1. Possible response to orientation and contrast of recurrent cortical networks with synaptic plasticity. (a) Left: Visual stimuli of orientation $\theta_{0}$ trigger input to neurons in the cortex. The amplitude of the input is larger to those neurons whose preferred orientation matches the stimulus orientation $\left(\theta=\theta_{0}\right.$, peak of curves). Visual contrast can also control the amplitude of the input to all neuronal preferred orientations (black, gray curves). Middle: Contrast-invariant neurons of different orientation preference respond selectively to tuned input. Through synapses, neurons additionally receive tuned recurrent input from other neurons with similar orientation preference to which they are preferentially connected. Right: The network response is a particular firing rate pattern that depends on the neuronal preferred orientation $\theta$, the stimulus orientation $\theta_{0}$ and contrast, and the state of the synapses. (b) Synaptic plasticity can modify the strength of recurrent connections following changes in the stimulus contrast. This controls the magnitude of the recurrent input to other neurons and can lead to different susceptibility to contrast in neurons with different preferred orientation $\theta$. (i) If contrast evenly rescales the firing rate by the same factor at all active orientation domains, the network selectivity is invariant to contrast. On the contrary, the network response becomes contrast-dependent if the firing rates at different orientations are unevenly rescaled by contrast: tuning width broadens with contrast (ii) if the nonpreferred orientations $\left(\theta \neq \theta_{0}\right)$ are more susceptible to increasing contrast or it narrows with contrast (iii) if the neurons with preferred orientation $\left(\theta=\theta_{0}\right)$ are more susceptible to increasing contrast. The type of rescaling by contrast (i, ii, or iii) is controlled at the synaptic level.

contrast-invariant neurons may not be invariant [16,17]. This is because synaptic plasticity can change the susceptibility to intensity in neurons depending on their feature preferences. But contrast invariance can only emerge if the representation of the stimulus is rescaled by the same factor upon changes in intensity at all active feature-selective neurons [Fig. 1(b), i]. Thus one could expect, in general, a recurrent network with synaptic plasticity to be contrast-dependent [Fig. 1(b), ii-iii]. To isolate how these plasticity-induced effects impact the emergence of intensity invariance, we consider network regimes in which excitation and inhibition are balanced $[21,22]$. This regime has been experimentally observed in cortices where invariant representations are present $[23,24]$. Theoretical studies have shown that in the limit of perfectly balanced networks the nonlinearity in the neuronal transfer function does not influence the activity of the network $[21,22]$. This leads to network responses that are linear functions of the input in the absence of synaptic plasticity $[21,22]$. Therefore one can assume that the nonlinearity in balanced circuits with synaptic plasticity is exclusive of synaptic origin [25]. For this reason we use the balanced network regime as a model system to study the impact of synaptic plasticity on the establishment of invariant representations. We used mean-field models and spiking network simulations [26].

We began by characterizing the type of nonlinearities that short-term plasticity (STP) - a ubiquitous form of cortical plasticity [15] — can introduce in balanced networks of randomly connected neurons. We then investigated these synaptic effects in networks with feature-dependent connectivity, a type of connectivity observed in cortical circuits [13,27]. For these types of networks, our results reveal how short-term plasticity can lead to intensity dependence by inducing different susceptibilities across the network. Yet we find that if the synaptic transformation approximates a power-law at the population level, this reconciles network nonlinearity and intensity invariance. We show how such a power-law-like approximation can be consistent with STP synapses. 
(a)

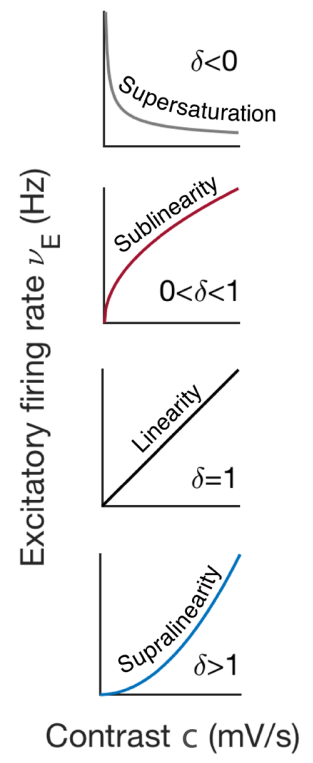

(b)

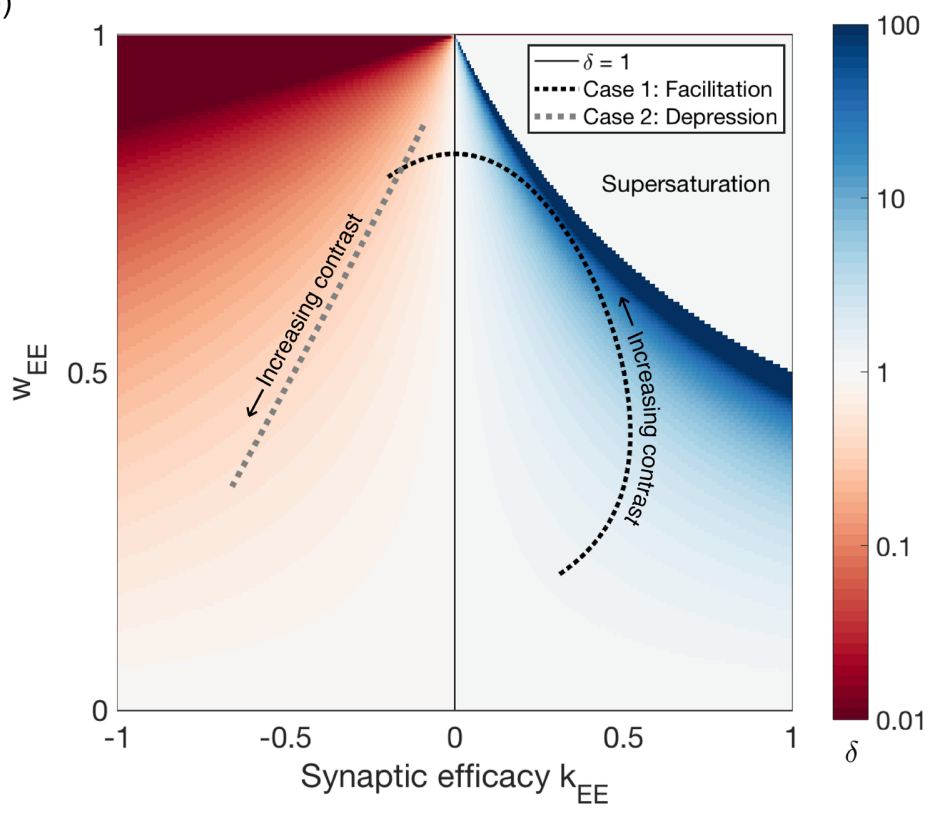

FIG. 2. Plastic $E \rightarrow E$ synapses control the susceptibility to input contrast $\delta$ in uniform balanced networks. (a) The contrast response characterized by the value of $\delta$ : supersaturating $(\delta<0)$, sublinear $(0<\delta<1)$, linear $(\delta=1)$, and supralinear $(\delta>1)$. (b) The phase space of values for $\delta$ [Eq. (2)] reveals a sublinear contrast response for depressing states $\left(k_{E E}<0\right)$ and a supralinear or supersaturating response for facilitating states $\left(k_{E E}>0\right)$. The network response is linear if synapses are constant $\left(k_{E E}=0\right.$, black line). The transition from the sublinear to the supralinear region is smooth, whereas the transition from the supralinear to the supersaturating is discontinuous and it is characterized by $\delta \rightarrow \infty$. The sublinear and supralinear nonlinearities are stable and can be dynamically reached by the network, whereas supersaturation is unstable and may not be realizable in balanced networks (see Supplemental Material for stability analysis [29]). The trajectories describe how $\delta$ evolves as a function of increasing input contrast in a rate network model with facilitating STP synapses (case 1) and in a network with depressing STP synapses (case 2) (see Methods - Model II and Table S1 for parameters [29]). Let us note that for positive firing rates and a stable balanced state, parameters must satisfy $w_{E E}<1$ [Eq. (3)] [21,22].

\section{RESULTS}

We examine how synaptic plasticity alone influences the network response to intensity in randomly connected balanced networks. We then investigate the effect of the intensity response on the emergence of invariant representations of sensory stimuli in balanced networks with feature-dependent connectivity. To that end we use rate models and spiking network simulations (see Methods for details). We examine four types of synaptic interactions: constant, short-term facilitating, short-term depressing [25], and power-law synapses (see Methods). The spiking network models that we examined consist of leaky integrate-and-fire (LIF) excitatory (E) and inhibitory (I) neurons. For illustration purposes, we present our results in the context of the visual system. Thus we refer to visual contrast as the intensity of the stimulus and to orientation as the feature of the stimulus that neurons code for.

\section{A. How synaptic plasticity modulates the network response to contrast}

Theoretical studies suggested that the nonlinearities in the contrast response can be explained through synaptic plasticity in balanced networks with uniformly random connectivity [25,28]. Here we examine which type of synaptic plasticity enables a particular response to contrast in this type of balanced networks. To that end we introduce the susceptibility to input contrast $\delta$ as an index. This is a measure for the relative change in the excitatory firing rate $v_{E}$ as a function of the input contrast $c$,

$$
\delta=\frac{d v_{E}}{d c} \frac{c}{v_{E}},
$$

where $\delta$ defines regimes of four types of contrast response functions [Fig. 2(a)]: supersaturation $(\delta<0)$, sublinearity $(0<\delta<1)$, linearity $(\delta=1)$, and supralinearity $(\delta>1)$. We assume excitatory input contrasts $(c>0)$.

In a balanced E/I network with synaptic plasticity in the $E \rightarrow E$ connections, uniform random connectivity and input contrast $c$ to the E and I populations [Eq. (10)], $\delta$ can be elegantly written as (see Methods for derivation)

$$
\delta=\frac{1-w_{E E}}{1-w_{E E}\left(1+\mathrm{k}_{E E}\right)} .
$$

Here, for simplicity, we have introduced the quantity $w_{E E}$, which denotes the normalized synaptic $E \rightarrow E$ strength for a particular firing rate:

$$
w_{E E}=\frac{J_{E E} J_{I I}}{J_{I E} J_{E I}} w_{E E}^{o}\left(v_{E}\right) .
$$

$J_{a b}$ are the constant weights for synapses connecting population $b$ to population $a$, where $a, b \in\{E, I\}$, and $w_{E E}^{o}$ is the firing-rate-dependent scaling factor of the $E \rightarrow E$ synapses. $w_{E E}^{o}$ can also be interpreted as the probability of neurotransmitter release in STP (see Supplemental Material for details on the STP model [29]). $\mathrm{k}_{E E}$ indicates how the synaptic 
strength changes with the firing rate:

$$
\mathrm{k}_{E E}=\frac{d w_{E E}}{d v_{E}} \frac{v_{E}}{w_{E E}} .
$$

The synaptic state is depressing if $\mathrm{k}_{E E}<0$ and facilitating if $\mathrm{k}_{E E}>0$. The phase space of values for $\delta$ [Fig. 2(b)] shows that depressing states $\left(\mathrm{k}_{E E}<0\right)$ lead to sublinear contrast response. Moreover, we find that supralinearity and supersaturation are only possible for facilitating synaptic states $\left(\mathrm{k}_{E E}>0\right)$. This is illustrated by the trajectories of the network response to increasing input contrast for networks with depressing and facilitating STP (Eq. S3 in the Supplemental Material [29]). The network with depressing synapses describes a trajectory in the sublinear space. The network with facilitating synapses occupies the supralinear space for positive values of $\mathrm{k}_{E E}$, while it transitions smoothly to the sublinear space as $w_{E E}$ saturates. A linear response only occurs if synapses are constant $\left(\mathrm{k}_{E E}=0\right)$. Additional $E \rightarrow I$ plasticity also permits the emergence of all types of nonlinearities (Supplemental Material, Fig. S2, Eq. S6 [29]).

There are two possible transitions between regimes. One is a smooth transition from the sublinear to the supralinear region (vertical black line at $\mathrm{k}_{E E}=0$ ). In this case $\delta$ varies continuously from values smaller than unity to values larger than unity. The other is a discontinuous transition from the supralinear regime to the supersaturating regime. This discontinuous transition is characterized by diverging network susceptibility $\delta \rightarrow \infty$. If a network approaches this transition from the supralinear regime, it will experience a rapid increase in firing rate. Facilitating short-term synapses cannot support this rapid increase in firing rate, leading to a transition of the trajectory to the sublinear regime. Network states in which $\delta<0$ are unstable (see Supplemental Material for stability analysis [29]). This result indicates that sublinearity, linearity, and supralinearity are realizable in balanced networks with uniform connectivity through plastic synapses. Supersaturation, however, is not supported in this type of network. In the next section we analyze the contrast response in a spiking network with uniform connectivity and STP.

\section{B. Susceptibility $\delta$ in spiking networks with uniform connectivity and STP}

In this section we study if the different types of contrast response functions reported in Fig. 2 emerge in a spiking network with uniformly random connectivity and $E \rightarrow E$ short-term synaptic plasticity (Eq. S3, Fig. S3 of the Supplemental Material [29]). STP modulates the synaptic strength as a function of the firing rate history of the presynaptic neuron [30] and acts on the timescale of milliseconds to seconds.

We examine the response to contrast in the spiking network model illustrated in Fig. 3(a) (see Model II in Methods). Spiking networks with STP facilitating or depressing $E \rightarrow E$ synapses have a nonlinear response to contrast [Fig. 3(b), dots]. The synaptic scaling factor $w_{E E}^{o}$ and the synaptic efficacy $\mathrm{k}_{E E}$ in these networks change with the firing rate [Figs. 3(d) and 3(e), dots]. In contrast, the network with constant synaptic weights is linear [Fig. 3(b), black dots, and Fig. S1 [29]]—-see Fig. S1 [29] for the firing rate of the inhibitory population when synapses are constant. We confirm that these networks behave in agreement with the analytical balanced state prediction [Eq. (10), Fig. 3, solid lines]. The coefficient of variation $\mathrm{CV} \approx 1$ values and exponentially decaying interspike interval (ISI) distributions [Fig. 3(c)] are consistent with in vivo recordings [31] and demonstrate that STP does not modify the irregularity in spiking activity (see Figs. S1c and S1d, Supplemental Material [29]).

We calculate $\delta$ in the spiking networks based on the values of $w_{E E}^{o}$ and $\mathrm{k}_{E E}$ displayed by plastic synapses [Fig. 3(f)]. As predicted, the spiking network with facilitating synapses exhibits a supralinear increase in firing rate $(\delta>1)$ that becomes sublinear $(0<\delta<1)$ for increasing contrasts [Fig. 3(f), blue]. This behavior is consistent with the facilitating $\delta$ trajectory in Fig. 2. For low firing rates, neurotransmitter is replenished fast enough in between spikes and facilitating synaptic transmission is sustained. The value of $\delta$ increases and the trajectory approaches the supersaturating unstable regime, where $\delta \rightarrow \infty$. This explains the peak in $\delta$ in Fig. 3(f). At this point, a further increase in synaptic strength cannot be physiologically supported because synapses demand neurotransmitter quicker than it can be produced. As a result, the network transitions smoothly from the supralinear to the sublinear regime. Conversely, the network with depressing synapses exhibits a sublinear increase in firing rate $(0<\delta<$ 1) for all the input contrasts analyzed, which is also in agreement with the trajectory in Fig. 2.

We show that short-term plasticity can have a dramatic effect on the nonlinear contrast response in spiking networks with uniformly random connectivity. In addition, we demonstrate that $\delta$ can be used as a tool to study the behavior of spiking networks with synaptic plasticity. Having singled out the effect of synaptic plasticity in a simple network topology, we now study how these nonlinearities affect the network selectivity to the stimulus orientation. Interestingly, a necessary condition for the balanced state is that the width of the input needs to be broader than the width of the recurrent connections (see also Eq. S30 [29], and [22]). To meet this criterion, we introduce orientation-dependent connectivity, which is a type of connectivity consistent with experimental data $[13,27]$.

\section{Facilitating $E \rightarrow E$ STP breaks contrast invariance in networks with orientation-dependent connectivity}

In this section we study whether the nonlinear contrast response induced by short-term synaptic plasticity is compatible with contrast invariance in networks with orientationdependent connectivity [see Model IV in Methods, Fig. 4(a)]. In the model [Fig. 4(a)] the neuronal population gets a Gaussian stimulus of orientation $\theta_{0}$ and tuning width $\sigma_{\text {stim }}$ [Eq. (11)] representing thalamocortical input (see Fig. S8 [29]). The stimulus contrast is a prefactor $c$ that scales the magnitude of the thalamocortical input at all orientations [10]. The probability of connection between neurons decays as a Gaussian with orientation distance such that neurons with a similar orientation preference are more likely to be connected, consistent with experimental data $[13,27]$. In addition, the $E \rightarrow E$ synaptic strength is modulated by short-term plasticity (see also Model IV in Methods). 
(a)

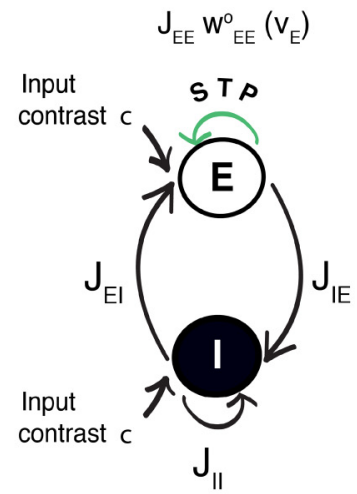

(d)

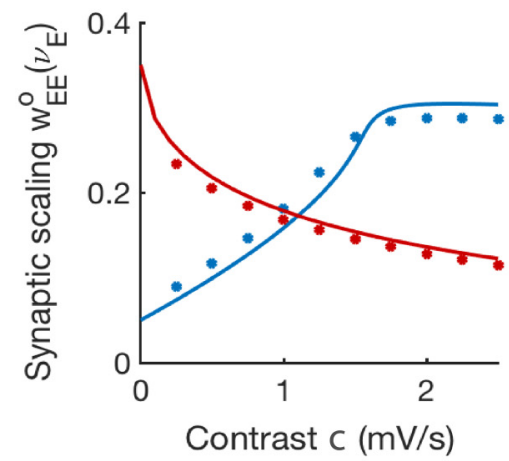

(b)

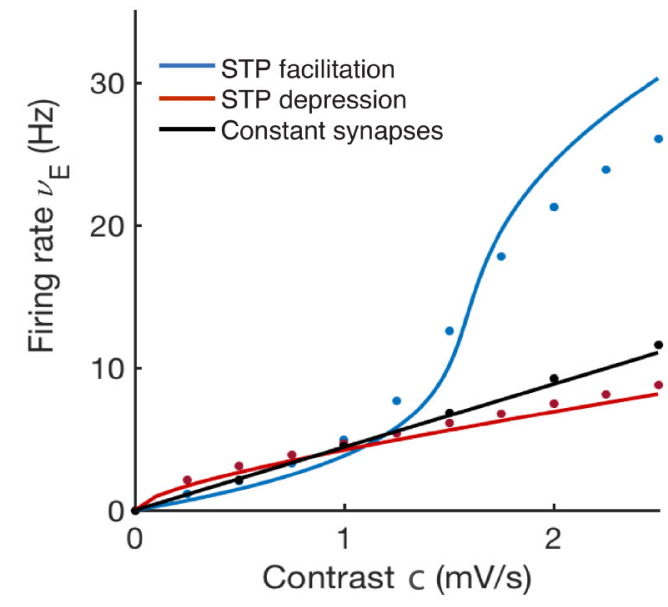

(e)

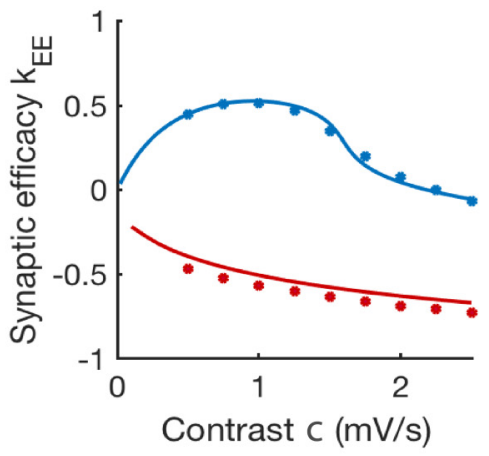

(c)

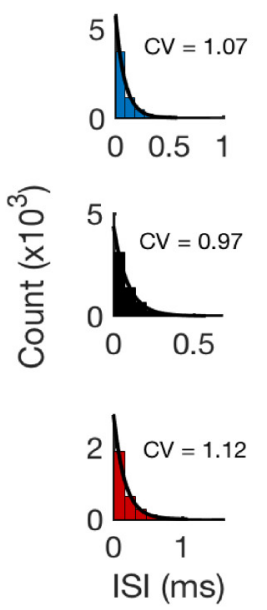

(c) (f)

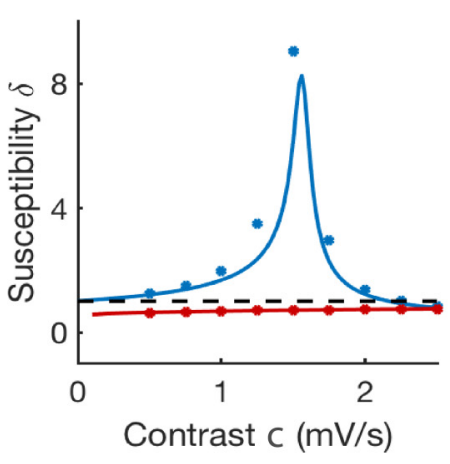

FIG. 3. Susceptibility to contrast $\delta$ in spiking networks of randomly connected neurons with $E \rightarrow E$ STP. (a) Uniformly randomly connected network (see Methods - Model II and Table S1 for parameters [29]). The $E \rightarrow E$ synaptic strength is modulated by the function $w_{E E}^{o}$, which describes the probability of neurotransmitter release as a function of the presynaptic firing rate (green arrow). Each neuron receives an external excitatory input that is directly proportional to the stimulus contrast $c>0$. (b) The firing rate of the $E$ population vs the input contrast for constant, facilitating, and depressing STP synapses. Data from spiking network simulations (dots) is shown alongside balanced network predictions from Eq. (10) (solid lines). (c) The coefficient of variation CV $\approx 1$, ISI histograms, and exponential fit denote Poissonian irregular firing patterns. Data computed over $1 \mathrm{~s}$ of spiking network simulation for an input contrast of $1.5 \mathrm{mV} / \mathrm{s}$. (d) The probability of neurotransmitter release $w_{E E}^{o}$ and (e) the synaptic efficacy $\mathrm{k}_{E E}$ in the two plastic networks studied. Predictions from the rate model (solid lines) are plotted alongside data from spiking network simulations (dots). (f) The susceptibility $\delta$ calculated from Eq. (2) (solid lines) and its value in spiking network simulations (dots). The dashed line indicates $\delta=1$.

Our results show that STP controls the nonlinearity in the response to contrast in networks with orientation-dependent connectivity. In this type of network, the response to contrast and the selectivity for orientation are related through (see Supplemental Material for the firing rate in networks with feature-dependent connectivity [29])

$$
v_{E}\left(w_{E E}-1\right) \propto c \frac{\sigma_{E}}{\sqrt{\sigma_{\text {stim }}^{2}-\sigma_{E}^{2}}} e^{-\frac{\left(\theta-\theta_{0}\right)^{2}}{2\left(\sigma_{\text {stim }}^{2}-\sigma_{E}^{2}\right)}} .
$$

Here $v_{E}$ denotes firing rate as a function of orientation, $w_{E E}$ is proportional to the probability of neurotransmitter release in STP, $c$ is the contrast of the stimulus, $\sigma_{\text {stim }}$ is the width of the thalamic input, and $\sigma_{E}$ is the width of the connectivity from $E \rightarrow E$ and from $E \rightarrow I$ neurons. Note that (1) networks with orientation-dependent connectivity and constant synapses are contrast-invariant, but this is just a consequence of the linearity of the balanced nonplastic network (Fig. S4 [29]), and (2) when the input is orientation dependent, the balanced state requires $\sigma_{\text {stim }}^{2}-\sigma_{E}^{2}>0$ for $v_{E}(\theta)$ to exist, which implies that networks of uniformly randomly connected neurons $\left(\sigma_{E} \rightarrow \infty\right)$ receiving orientation-dependent input do not have a balanced $v_{E}(\theta)$ solution [22] [Eq. (5)].

We show in Fig. 4 results for a network with orientationdependent connectivity and STP facilitating $E \rightarrow E$ synapses. The solution of Eq. (5) for these network parameters is given in Fig. 4(b) (solid lines) for several input contrasts $c$. When synapses are facilitating, increasing the stimulus contrast $c$ does not linearly rescale $v_{E}$. This effect is also present in spiking networks [Fig. 4(b), dots]. We quantify the contrast dependence on selectivity using the full width at half maximum (FWHM) [Fig. 4(c), top]. With an increase in contrast, the FWHM first decreases, 
(a)

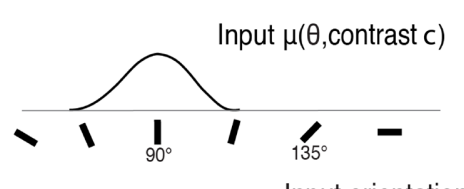

$p_{E}(\Psi-\theta)$

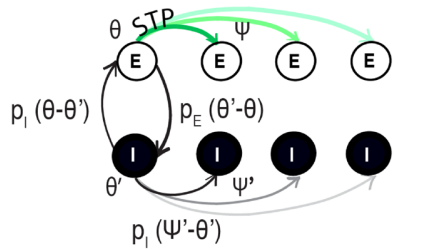

High probability Low probability (b)

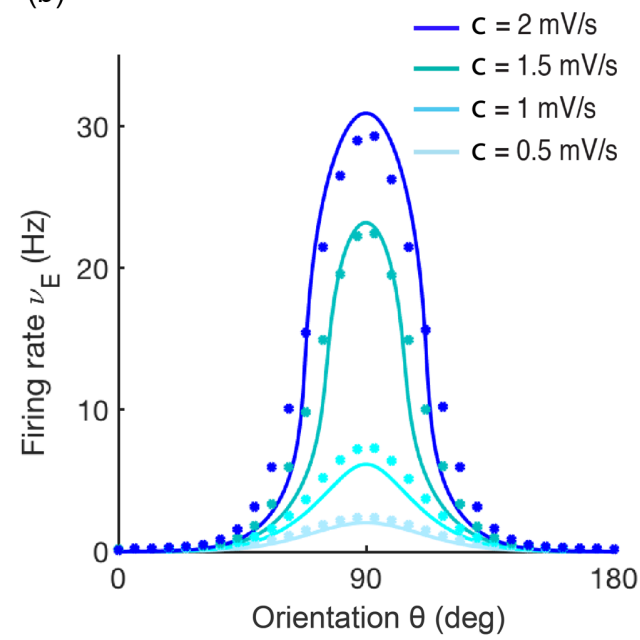

(c)
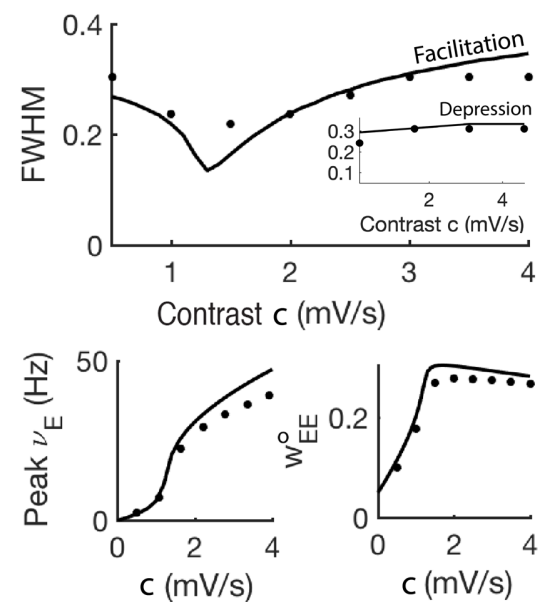

FIG. 4. Facilitating $E \rightarrow E$ STP yields nonlinear susceptibility to input contrast and breaks contrast invariance (see Fig. S5 for depressing $E \rightarrow E$ synapses). (a) Network with orientation-dependent connectivity and $E \rightarrow E$ STP synapses (see Methods - Model IV and Table S1 for parameters [29]). (b) Excitatory tuning curves for different input contrast, $c=0.5,1,1.5,2$ (mV/s). Predictions from the balanced theory [Eq. (5), solid lines] compared to the results obtained in a simulation of spiking neurons (dots). (c) Upper panel: FWHM changes with contrast, which confirms contrast-dependent selectivity. Inset: comparison to a quasi-invariant network with $E \rightarrow E$ depressing synapses (see Fig. S5 for depressing tuning curves [29]). Lower panel: Peak firing rate increases supralinearly until $c$ approaches $1.5 \mathrm{mV} / \mathrm{s}$ (left), which is consistent with the increase in the probability of neurotransmitter release $w_{E E}^{o}$ (right). As a signature of facilitating transmission, when firing rates continue to increase for $c>1.5 \mathrm{mV} / \mathrm{s}$, the probability of neurotransmitter release $w_{E E}^{o}$ decreases (right) and the network behavior becomes sublinear (left). The shift from supralinearity to sublinearity is consistent with the trajectory in Fig. 2 (case 1).

indicating a narrowing. Interestingly, after reaching a minimum the FWHM then increases, revealing a subsequent broadening. This dependency on contrast is consistent with the facilitating trajectory in Fig. 2. For a given input contrast, the neurons tuned to the stimulus orientation $\theta_{0}$ receive higher input than any other position in the network, and the firing rate increases. As a result the point of maximum supralinearity is approached and the value of $\delta$ is higher than the one at positions that have a different preferred orientation, which narrows the selectivity. If at this point the contrast increases, synapses at the position tuned to the stimulus orientation can no longer meet the demand for neurotransmitter required to sustain high firing rates, which causes a decrease in $\delta$. As the position tuned to the stimulus orientation approaches sublinearity, positions tuned for the nonpreferred orientations approach the point of maximum supralinearity, which broadens the selectivity (see Video S1 [29]). The transition between nonlinear $\delta$ regimes is also seen in the network response to contrast and in the STP probability of neurotransmitter release [Fig. 4(c), bottom, for $\theta=\theta_{0}$ ]. These results demonstrate that the nonlinear contrast response induced by facilitating $E \rightarrow E$ STP can lead to contrast-dependent orientation selectivity. We repeat this analysis for networks with purely depressing $E \rightarrow E$ synapses (Fig. S5 [29]). In this case our results show that pure $E \rightarrow E$ STP depression induces sublinearity and quasi-contrast-invariant selectivity (Fig. S5b-c [29]) for the STP parameters analyzed. This is demonstrated by the overlapping tuning curves in Fig. S5c [29] and suggests that depressing $E \rightarrow E \mathrm{STP}$ does not induce large changes in the susceptibility to input contrast across orientation domains in the network studied. In the next section we analyze what is it that preserves a quasi-invariant selectivity when synapses are STP depressing - as opposed to facilitation-and if it is possible for a network with facilitating synapses to be contrast-invariant.

\section{A power-law synaptic transformation is required for contrast invariance in networks with orientation-dependent connectivity}

In this section we show that the reconciliation between the nonlinear contrast response, facilitating and depressing STP, and the emergence of contrast invariance is plausible under certain synaptic conditions. Previous theoretical studies have reported that only a power-law function can transform contrast-invariant membrane potential input into contrastinvariant firing rate output in single neurons [32,33]. If single neurons transformed intracellular membrane potential into firing rate through a linear-threshold function they would exhibit a broadening of orientation tuning at high contrast. This is because the responses to nonpreferred orientations reach the spiking threshold as contrast is increased [34]. A power-law nonlinearity, however, rescales firing rates with contrast by the same amount at all orientations, which preserves contrast invariance [32,33]. We test an analogous requirement for synapses at the network level and impose a power-law synaptic transformation at the $E \rightarrow E$ and $E \rightarrow I$ populations, $w_{E E}=w_{I E} \sim v_{E}^{\mathrm{k}}$ (see Model V in Methods). Note that both a power-law transformation in the $E \rightarrow E$ [Eq. (5)] and STP in the $E \rightarrow E$ and $E \rightarrow I$ connections (Fig. S6 [29]) can lead to contrast dependence. Instead, here the response to contrast and the response to orientation are independent from each other, which yields the network selectivity $v_{E}$ invariant to contrast (see Supplemental Material for the firing rate in 
(a)

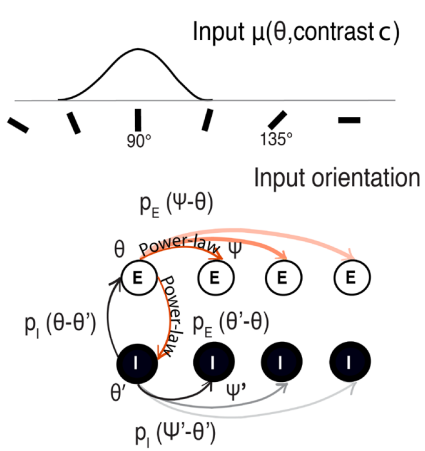

High probability Low probability (b)

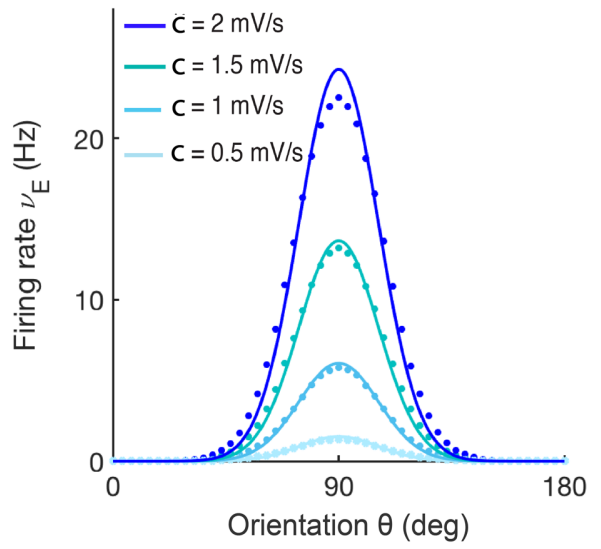

(c)

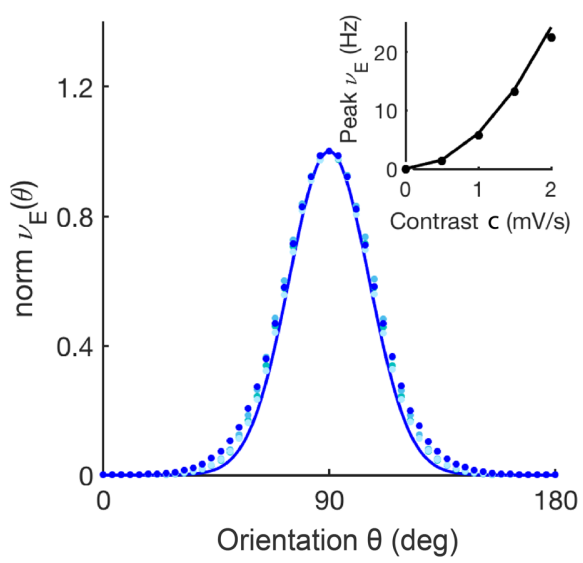

FIG. 5. Networks with orientation-dependent connectivity and power-law $E \rightarrow E$ and $E \rightarrow I$ synapses show nonlinear susceptibility and contrast-invariant tuning. (a) Network with orientation-dependent connectivity. Additionally, in this network the $E \rightarrow E$ and $E \rightarrow I$ synapses are nonlinearly modulated by the function $w_{E E}=w_{E I} \sim v_{E}^{\mathrm{k}}$, where $\mathrm{k}$ is the synaptic efficacy (red arrows) (see Methods - Model V and Table $\mathrm{S} 1$ for parameters [29]). (b). Excitatory tuning curves in response to several input contrast $c=0.5,1,1.5,2$ (mV/s). Predictions from the analytical balanced state [Eq. (6), solid lines] are compared to the results obtained in a simulation of spiking neurons (dots). (c) Normalized analytical (solid lines) and simulation tuning curves (dots) from (b) show that this type of network is contrast invariant. Inset: Peak excitatory firing rate at $\theta=90^{\circ}$ increases supralinearly with contrast in the analytical balanced state (solid line) as well as in spiking network simulations (dots).

networks with feature-dependent connectivity [29]):

$$
v_{E} \propto c^{\frac{1}{1+\mathrm{k}}}\left(\frac{\sigma_{E}}{\sqrt{\sigma_{\mathrm{stim}}^{2}-\sigma_{E}^{2}}} e^{-\frac{\left(\theta-\theta_{0}\right)^{2}}{2\left(\sigma_{\mathrm{stim}}^{2}-\sigma_{E}^{2}\right)}}\right)^{\frac{1}{1+\mathrm{k}}} .
$$

In Eq. (6) the synaptic efficacy $\mathrm{k}$ is independent of the presynaptic firing rate. In this type of network, the susceptibility $\delta$ is given by $\delta=\frac{1}{1+\mathrm{k}}$. The network response is sublinear if $\mathrm{k}>0$, linear if $\mathrm{k}=0$, and supralinear if $-1 \leqslant \mathrm{k}<0$. The analytical solution of Eq. (6) for $\mathrm{k}=-0.5$ is given in Fig. 5(b) (solid lines) for several input contrasts $c$. We start by numerically validating Eq. (6) in spiking networks in which the probability of neurotransmitter release at individual $E \rightarrow E$ and $E \rightarrow I$ synapses follows a power-law (see Model $\mathrm{V}$ in Methods). Figure 5(b) shows that the activity of a spiking network with orientation-dependent connectivity and power-law synapses can be predicted by Eq. (6). It is possible to factorize Eq. (6) into a function of contrast and a function of orientation, which indicates contrast invariance. The normalization of the network activity demonstrates that contrast invariance is also present in spiking networks [Fig. 5(c), dots]. As predicted, the contrast response is supralinear with $\delta=2$ [Fig. 5(c), inset for $\left.\theta=\theta_{0}\right]$. This demonstrates that depressing synaptic states can lead to a supralinear response to contrast in spiking networks [Fig. 5(c)].

Equation (6) confirms that both facilitating $(\mathrm{k}>0)$ or depressing $(\mathrm{k}<0)$ power-law synaptic models support the emergence of perfect contrast invariance and a nonlinear response to contrast in spiking networks with orientationdependent connectivity. However, a power-law at each individual synapse is not a necessary condition for invariance [Eq. (6), see Methods for mean-field derivation]. Instead,
Eq. (6) demonstrates that the averaged transformation performed by a population of synapses needs to follow a power-law rather than each individual synapse. Indeed, individual synapses in invariant networks do not necessarily behave as power-laws. This is particularly notable in the case of facilitating STP (Fig. S3 [29]), but also true for purely depressing STP synapses, which may not approximate power-law transformations at low firing rate values (see Fig. S6c [29]). In line with this, we investigate ways to approximate such power-law transformations that are compatible with the physiology of facilitating and depressing STP synapses.

We found that the requirement of a power-law transformation can be approximated by combining facilitating and depressing STP synapses. To show this we split the excitatory synaptic population into two subpopulations, each of them having different STP dynamics [Fig. 6(a): one facilitating $\left(\mathrm{E}_{\mathrm{fac}}\right)$ and one depressing $\left.\left(\mathrm{E}_{\mathrm{dep}}\right)\right]$. On average, i.e., $\left(\mathrm{w}_{\mathrm{E}}^{o}=\right.$ $\left.\alpha \mathrm{w}_{\mathrm{fac}}^{o}+[1-\alpha] \mathrm{w}_{\mathrm{dep}}^{o}\right)$, the synaptic transformation performed by the excitatory population approximates a power-law function of the presynaptic firing rate, where $\alpha$ is the fraction of the excitatory population having facilitating synapses [Fig. 6(a)]. We show that the combination of facilitation and depression approximates a power-law (Fig. 6) and that the fit is better for low presynaptic firing rates values (Fig. S7, black [29]) compared to pure depression (Fig. S7, blue [29]). We implement this idea in a spiking network where individual $E \rightarrow E$ and $E \rightarrow I$ synapses follow STP [Figs. 6(b) and 6(c)]. The response of the network to a stimulus presented at different contrasts $c$ is given in Fig. 6(b) (dots) alongside the mean-field results (solid lines). The data plotted in Fig. 6(b) show that the spiking network and the mean-field model results are consistent. We quantify the degree of invariance using the FWHM. Our results show that the FWHM is approximately constant 
(a)
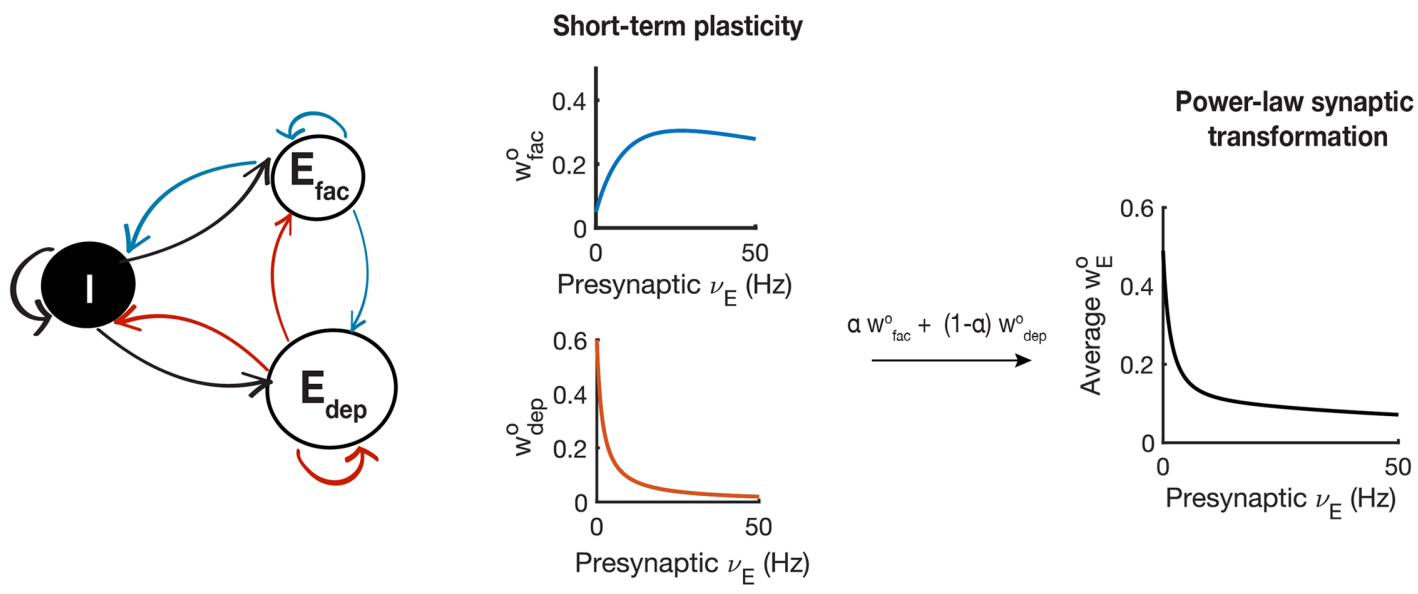

(b)

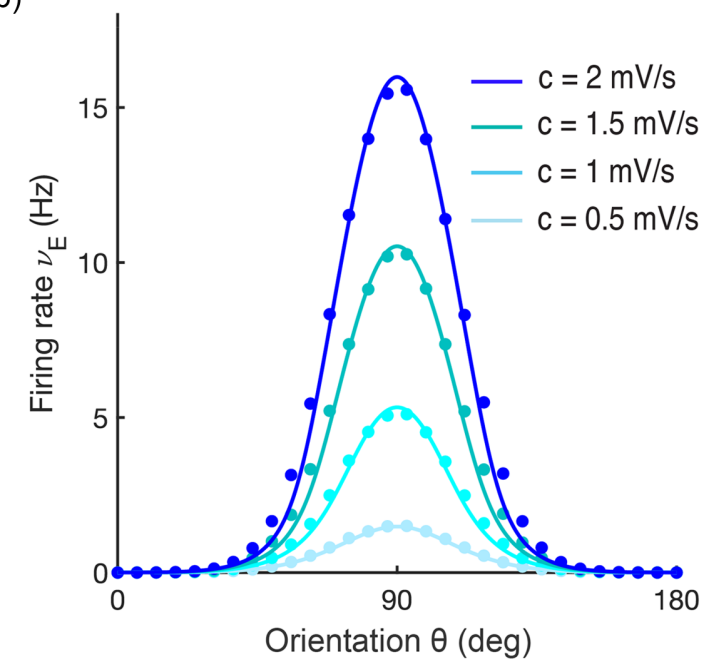

(c)
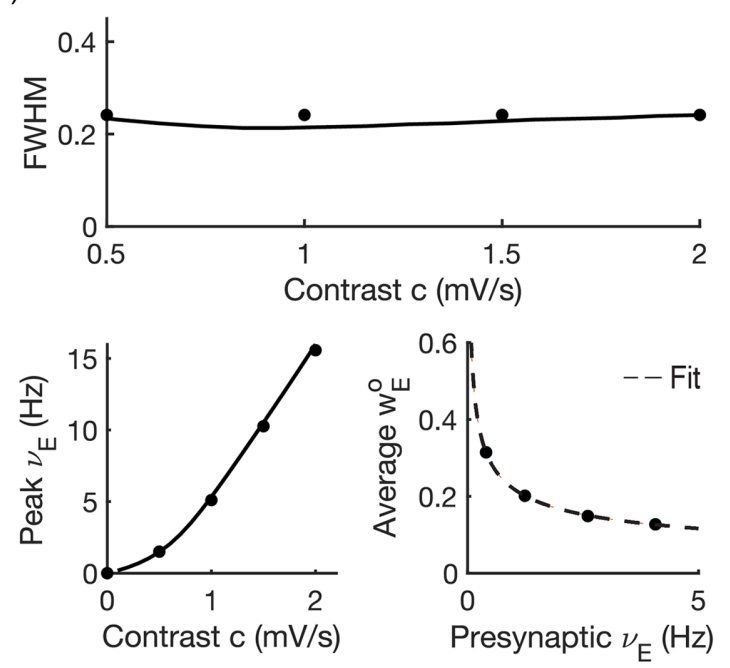

FIG. 6. Networks of two excitatory subpopulations with $E \rightarrow E$ and $E \rightarrow I$ STP can approximate contrast invariance. (a) Network diagram (left) and average STP synaptic transformation (right). The synapses of the excitatory subpopulation $\left(\mathrm{E}_{\text {fac }}\right)$ follow facilitating STP ( $w_{\text {fac }}^{o}$ ). The synapses of the excitatory subpopulation $\left(\mathrm{E}_{\mathrm{dep}}\right)$ are depressing and their release probability is given by $w_{\mathrm{dep}}^{o}$. On the right, the average neurotransmitter release probability of the excitatory population $\left(\mathrm{w}_{E}^{o}=\alpha \mathrm{w}_{\mathrm{fac}}^{o}+[1-\alpha] \mathrm{w}_{\mathrm{dep}}^{o}\right)$, where $\alpha=0.2$ is the fraction of the population in a facilitating state. The value of $\alpha$ is chosen such that $w_{E}^{o}$ approximates a power-law for the STP parameters given in Table S1 [29]. Notice that orientation-dependent connectivity is not shown in the network diagram (see Methods - Model VI and Table S1 for parameters [29]). (b) Tuning curves of the network in response to a stimulus of orientation $\theta_{0}=90^{\circ}$ at different contrasts $c=0.5,1,1.5,2 \mathrm{mV} / \mathrm{s}$. The spiking network simulation results (dots) are shown alongside the solution of the rate model. (c) Top: FWHM for the contrasts $c$ analyzed in (b). Bottom left: Firing rate at the preferred orientation $\left(\theta=90^{\circ}\right)$ as a function of contrast. Bottom right: Average neurotransmitter release probability $\mathrm{w}_{\mathrm{E}}^{o}$ as a function of contrast in the spiking network simulation (dots) and fit to a power-law (dashed line). Fit $=a v_{E}^{k}(a=0.2758$ and $k=-0.3494)$.

across all contrast input levels analyzed, which confirms contrast invariance [Fig. 6(c), top]. We also show that the firing rate at the preferred orientation $\left(\theta=\theta_{0}\right)$ scales supralinearly with contrast [Fig. 6(c), bottom left], which is a consequence of the nonlinear STP dynamics [Fig. 6(c), bottom right]. These results show that the power-law transformation required for contrast invariance and a nonlinear response to contrast is compatible with the type of STP dynamics observed in cortical invariant circuits.

\section{DISCUSSION}

We analyzed the interplay between synaptic plasticity, network response to stimulus contrast, and selectivity for the stimulus orientation. Counterintuitively, in balanced networks contrast invariance of the network does not necessarily follow from the contrast invariance of individual neurons. Here we showed how for balanced networks the synaptic state controls the nonlinearity in the network response to contrast (Figs. 2 and 3), from sub- to supralinear. Moreover, our results show that synaptic plasticity modulates feature selectivity and leads to contrast-dependent (Fig. 4, S6 [29]) or contrast-invariant (Figs. 5 and 6) network selectivity. Contrast-invariant selectivity requires an effective power-law synaptic transformation that is compatible with STP (Fig. 6). Therefore this proposes a mechanism to reconcile a nonlinear contrast response, STP, and contrast invariance at the network level that is compatible with $\mathrm{E} / \mathrm{I}$ balance. 


\section{A. Network response to contrast}

In this work we propose a measure to describe the nonlinear behavior of the network activity and its dependence on its synaptic states: the susceptibility to contrast $\delta$ [Eq. (2)]. The susceptibility $\delta$ captures the relative changes in the excitatory firing rate with contrast. In balanced networks with uniform random connectivity and $E \rightarrow E$ plastic synapses, we show that the susceptibility to contrast $\delta$ depends only on two variables: the effective synaptic strength $w_{E E}$ and its relative dependence on firing rate $v_{E}$, which we characterize by $\mathrm{k}_{E E}$ [see Eq. (4)]. Using the concept of susceptibility $\delta$, we identified four types of contrast responses: supralinear, linear, sublinear, and supersaturating (Fig. 2). The phase space of $\delta$ values allows to connect the synaptic state to phase transitions of network behavior (Fig. 2, trajectories). We show trajectories spanning the broad phase space of nonlinear responses for uniform spiking networks with facilitating and depressing STP (Fig. 3). We also show that a supersaturating nonlinearity is unstable in balanced networks with plastic $E \rightarrow E$ synapses (see Supplemental Material for stability analysis [29]).

These results apply to networks with E/I balance. Previous theoretical studies have shown that non-neuronal mechanisms like synaptic plasticity are necessary to achieve a nonlinear response in balanced networks [25]. On the experimental side, studies have reported the cancellation of E/I currents in cortices where contrast invariance is present [23]. Furthermore, $\mathrm{E} / \mathrm{I}$ balance has been proposed as a necessary mechanism for the emergence of sharp orientation selectivity in neurons located at pinwheels in V1 [24]. For completeness let us note that when excitation and inhibition are loosely balanced [35], the neuronal nonlinearity has been shown to shape the network response to contrast. This scenario is captured by the supralinear stabilized network model (SSN), which considers constant synapses [36,37]. The SSN permits supersaturation in rate models [36,37] and in spiking networks [38]. Here, to single out the synaptic effect on the network nonlinearities we considered balanced E/I networks with plastic synapses.

\section{B. Contrast-dependent selectivity}

In networks with orientation-dependent connectivity, for constant synapses the response to contrast is linear and the network is contrast invariant (Fig. S4 [29]). This is a consequence of the linearity of the network for all orientations $\theta$. However, when synapses follow STP plasticity (Eq. S3 [29]), the response to contrast is nonlinear and the network may become contrast dependent (Fig. 4, S6 [29]).

We found that facilitating $E \rightarrow E$ STP states can narrow the network selectivity (Fig. 4), while depressing $E \rightarrow E$ states leads to a quasi-invariance that only slightly broadens the selectivity (Fig. S5 [29]). The change in selectivity induced by facilitating $E \rightarrow E \mathrm{STP}$ synapses can be explained as follows: A stimulus of orientation $\theta_{0}$ preferentially depolarizes some neurons in the network. As a consequence, the firing rate $v_{E}\left(\theta_{0}\right)$ of these neurons increases. This increase activates synapses, which start to release neurotransmitter with probability $w_{E E}^{o}\left(v_{E}\left(\theta_{0}\right)\right)$. Conversely, neurons tuned to nonpreferred orientations $\psi$ hardly receive external input and $v_{E}(\psi)<v_{E}\left(\theta_{0}\right)$, and their synapses release neurotransmitter with probability $w_{E E}^{o}\left(v_{E}(\psi)\right)$. When the stimulus contrast increases, $v_{E}\left(\theta_{0}\right)$ and $v_{E}(\psi)$ increase, and the probability of neurotransmitter release changes at both orientations. The key to understanding this phenomenon is that in STP, the relative change in release probability depends on the starting firing rate value (Eq. S3 [29]). Thus the synaptic efficacy $\mathrm{k}_{E E}\left(\theta_{0}\right) \neq \mathrm{k}_{E E}(\psi)$ [Eq. (4)]. As a result, if the synaptic state is facilitating, the firing rate at the network preferred orientation may be more supralinearly amplified compared to the orthogonal orientation. In this case the network selectivity narrows. Conversely, if the synaptic state is depressing, the firing rate at the preferred orientation is dampened compared to the nonpreferred orientations, which could potentially broaden the selectivity (see Video S1 [29]). Importantly, in depressing STP, however, the probability of neurotransmitter release approximates a power-law function for medium-to-high firing rate values, which leads to less pronounced changes in selectivity as a function of contrast (Fig. S3 [29]). Contrast dependence has been observed in the auditory cortex, where the network selectivity for the frequency of a tone changes when measured at different sound intensity levels [12,39-42]. Therefore our results suggest that facilitating STP may play an important role in modulating the network selectivity as a function of contrast in these areas.

\section{Contrast-invariant selectivity}

The aforementioned scenario changes if the synaptic strength has a power-law-like dependence as a function of firing rate [Eq. (6)]. In this scenario we found that a nonlinear response to contrast is consistent with contrast invariance in balanced networks with orientation-dependent connectivity, including positive (facilitating) or negative (depressing) exponents. We showed that this can be achieved by networks with perfect power-law excitatory synapses (Fig. 5) and approximated by networks with depressing $E \rightarrow E$ STP synapses (Fig. S5 [29]) or networks with a combination of facilitating and depressing excitatory STP (Fig. 6). Thus contrast invariance does not require that the synaptic strength of each synaptic terminal has to scale as a power-law function of the presynaptic firing rate, which would be unlikely due to the heterogeneity observed across synaptic terminals [43] and the widespread evidence of STP in cortical synapses [15]. Instead, it is the average synaptic strength of a given population that should scale as a power-law for a circuit to be contrast invariant. This condition must be fulfilled, at least, for excitatory synapses, regardless of whether the postsynaptic neuron is excitatory or inhibitory. Why do population-wide power-law synaptic transformations permit a nonlinear response to contrast and contrast-invariant selectivity while other synaptic plasticity rules do not (i.e., purely facilitating STP)? Previous studies suggest that a power-law is the only function that can transform contrast-invariant input into contrast-invariant spike output [33]. This is because power-law functions are scale-free transformations. In other words, a power-law-like synapse will amplify or compress the presynaptic rate by the same relative amount, independently of the firing rate of the presynaptic neuron. This ensures that the susceptibility $\delta$ is the same across orientations and for all firing rates. This universal nonlinear rescaling of the network activity supports contrast-invariant selectivity and permits the nonlinearity in 
the response to contrast. Power-law transformations have been previously reported in the cortex at the neuronal level $[20,44]$. Experiments showed that this type of transformation in single neurons can result from neuronal noise, which smooths the linear-threshold neuronal transfer function into a power-law $[20,44]$. In agreement with this, theoretical studies demonstrate that a power-law transformation of membrane potential input into firing rate output is a requirement for single-neuron contrast invariance [32,33]. However, a power-law neuronal nonlinearity is insufficient to explain contrast invariance at the circuit level [16] in balanced networks. This is because in balanced networks the averaged activity is independent of the neuronal properties [21]. If that is the case and if a powerlaw transformation is necessary for the emergence of contrast invariance at the network level, then the power-law should be supported by synaptic rather than by neuronal mechanisms in balanced networks. At the circuit level, a power-law transformation can also be approximated by an averaging mechanism across synapses that release neurotransmitter following distinct facilitating and depressing STP dynamics. We show this behavior for a two-excitatory population network (Fig. 6), but the concept is applicable to networks with larger synaptic variability. This suggests an analogy for the emergence of power-laws in single neurons and in networks: the former emerges from the neuronal variability in spiking responses [20] while the latter could be the result of synaptic variability across populations. If that were the case, it would be possible that neuronal and synaptic power-law nonlinearities could coexist, each of them serving the function of contrast invariance at a different level. For example, contrast-invariant selectivity in single neurons and at the circuit level is found in the visual cortex $[16,45]$ and the piriform cortex [6]. In the piriform cortex, the selectivity for a particular odorant is not modified by the concentration of odorant molecules in the air. In addition, our results suggest that the emergence of contrast invariance or contrast dependence in cortical circuits may not require major anatomical differences. Indeed, numerous experiments report a similar columnar structure, the same number of cortical layers, similar cell types, and similar inputoutput organization across sensory cortices [46,47]. Instead, our results suggest that these differences in selectivity across auditory, visual, and piriform cortices could be explained through synaptic physiology.

\section{Biological plausibility}

Our results predict that the excitatory synaptic strengths of neuronal populations in contrast-invariant cortices should scale as a power-law with the excitatory presynaptic firing rate. This scaling is consistent-for a broad range of firing rates - with the depressing STP-modulated amplitude of the postsynaptic current as a function of presynaptic firing rates measured in patch clamp recordings from pairs of neocortical pyramidal neurons [48] and in local field potentials in slices of rat visual cortex [49]. But our analysis also reveals that such power-law synaptic transformations could emerge from a combination of facilitating and depressing STP (Fig. S7 [29]). Moreover, our results suggest there is a dependency between short-term plasticity and the network selectivity (Figs. 4-6). To show experimental evidence of this dependency it would be necessary to assess the impact of the synaptic strength modulation on the network firing rate as a function of contrast. But how can one assess the impact of the synaptic strength-which is a dynamic and distributed cellular property acting at the microscale-on the overall behavior of the network such as its firing rate? Given that the synaptic strength is sensitive to variations in the extracellular fluid, spontaneous activity, patterns of stimulation, and the presence of neuromodulators [50], it is important to study in vivo data. However, this is challenging. For example, one needs to identify cellular connections. One way could be to identify synaptically connected neurons guided either by single-cell optogenetic control of the presynaptic activity [51] or by fluorescent genetic labeling of specific cell types [52,53]. Once synaptic strengths can be measured in a representative number of synaptic connections, differences in the synaptic physiology of invariant and noninvariant cortices can be studied, as our theoretical results suggest. In a next step, neuromodulators could be employed, which can change the function and dynamics of synapses and circuits [54], while the network activity could be recorded using two-photon calcium imaging. Indeed, the application of cholinergic agonists or antagonists has been shown to change the receptive field properties of single neurons in the somatosensory [55] and auditory cortex in vivo [56]. As a neuromodulator, one promising candidate is adenosine: First, it down-regulates the release probability at excitatory synapses, while it has no or little effect on inhibitory transmission [57,58]. Second, the mechanism mediating the decrease in release probability is presynaptic (as in our model): the activation of $\mathrm{A} 1$ adenosin receptors reduces the open probability of presynaptic $\mathrm{Ca}^{2+}$ channels [58]. Our results predict that contrast-invariant cortices loose this property when the synaptic behavior is disrupted by the administration of neuromodulators, while contrastdependent cortices may experience changes in their selectivity properties.

In summary, we have shown that different types of synaptic plasticity can generate a variety of nonlinearities in the representation of input contrast. These play a crucial role in the establishment of selectivity in balanced networks, where contrast invariance requires a power-law-like synaptic transformation that is compatible with STP. Thus our results indicate that the ability of balanced cortical networks to extract invariant information about sensory stimuli is directly connected to the physiology of synapses.

\section{START METHODS}

\section{A. Detailed description of the spiking network implementation}

We study six network models (I, II, III, IV, V, and VI) [26]. All the models consist of $N$ neurons, of which $N_{E}=$ $q N$ are excitatory and $N_{I}=(1-q) N$ are inhibitory, where $q=0.8$ is the fraction of excitatory neurons [59]. Neurons are uniformly distributed in a one-dimensional state space of orientation preference. Let us remark that this defines the feature space, not the physical space. Neuron $i$ in population $a$ has the orientation preference $\theta=\frac{i}{N_{a}}\left(i=1, \ldots, N_{a}\right)$, where $a=$ $\{E, I\}$. Connected neurons with preferred orientation $\theta$ and $\psi$ are sampled from a probability distribution $\sim p_{a b}(\theta-\psi)$. 
We consider a periodic domain $\Gamma=[0,1]$ such that on $\Gamma$, $p_{a b}^{\Gamma}(\theta-\psi)=\sum_{m=-\infty}^{\infty} p_{a b}(\theta-\psi-m)$. Let us note that in the figures we rescale $\Gamma$ to $180^{\circ}$ for illustrative purposes. We assume $p_{E E}=p_{I E}=p_{E}$ and $p_{E I}=p_{I I}=p_{I}$. The membrane potential $V_{i}^{a}(t)$ of neuron $i$ from population $a=\{E, I\}$ obeys the leaky-integrate-and-fire (LIF) dynamics described by

$$
\frac{d V_{i}^{a}}{d t}=-\frac{1}{\tau_{m}}\left(V_{i}^{a}-V_{\text {rest }}\right)+\frac{R_{m} I_{i}^{a}}{\tau_{m}},
$$

where $\tau_{m}$ is the membrane time constant, $V_{\text {rest }}$ is the resting potential, $R_{m}$ is the membrane resistance, and $I_{i}^{a}(t)$ is the sum of the recurrent current from other neurons in the network and an external feed-forward current $F_{\mathrm{ffw}}(\theta)$. Whenever $V_{i}^{a}(t)$ reaches the threshold voltage $V_{t h}$, neuron $i$ spikes and its membrane potential is reset to $V_{\text {rest }}$. Each neuron in the network receives input from a fixed number of $C_{E}$ and $C_{I}$ presynaptic excitatory and inhibitory neurons, respectively. The total synaptic input to the $i$ th neuron from population $a=\{E, I\}$ is given by

$$
\begin{aligned}
\frac{R_{m} I_{i}^{a}}{\tau_{m}}= & \sum_{j=1}^{N_{E}} \sum_{n} \frac{J_{a E}^{i j}}{\sqrt{N}} w_{a E, n}^{0, i j} \delta\left(t-t_{n}{ }^{b, j}\right) \\
& -\sum_{j=1}^{N_{I}} \sum_{n} \frac{J_{a I}^{i j}}{\sqrt{N}} w_{a I, n}^{0, i j} \delta\left(t-t_{n}{ }^{b, j}\right)+F_{\mathrm{ffw}}(\theta),
\end{aligned}
$$

where $\sum_{n} \delta\left(t-t_{n}{ }^{b, j}\right)$ is the spike train of the $j$ th neuron from population $b, J_{a b, 0}^{i j} / \sqrt{N}$ is the maximal synaptic weight from neuron $j$ in population $b$ to neuron $i$ in population $a$, and $w_{a b, n}^{0, i j}$ is a synaptic plasticity factor, which here depends on the spike time. If two neurons are connected, $J_{a b}^{i j}=J_{a b}$; otherwise $J_{a b}^{i j}$ is zero. Given that each connection is rescaled by $\frac{1}{\sqrt{N}}$, the total recurrent input is on the order of $\sqrt{N}$. The feed-forward input is given by $F_{\mathrm{ffw}}(\theta)=\sqrt{N} \mu+\sigma_{\theta} \xi$. Here, $\mu=c \cdot \bar{\mu}(\theta)$, where $c \geqslant 0$ denotes contrast, $\bar{\mu}(\theta)$ is the orientation-dependent component of the input, and $\xi$ is white noise with standard deviation $\sigma_{\theta}$. All parameters are given in Table S1 [29].

\section{B. Methods related to the rate formalism}

The firing rate of population $a=\{E, I\}$ is given by $v_{a}(\theta) \equiv$ $\left[\left\langle s_{a, j}(t)\right\rangle\right]$, where $s_{a, j}(t)=\sum_{n} \delta\left(t-t_{n}{ }^{a, j}\right)$ is the spike train of the $j$ th neuron from population $a,\langle\cdot\rangle$ denotes temporal average, and [.] denotes population average. Assuming E/I balance $[21,22,25]$, the mean input currents are related to the firing rates as

$$
\begin{aligned}
\mu_{a}(\theta) \equiv & \left\langle\left[I_{i}^{a}(\theta, t)\right]\right\rangle=\sqrt{N}\left[J_{a E} \frac{N_{E}}{N} p_{a E} *\left(w_{a E}^{o} v_{E}\right)\right. \\
& \left.-J_{a I} \frac{N_{I}}{N} p_{a I} *\left(w_{a I}^{o} v_{I}\right)+\mu\right],
\end{aligned}
$$

where $*$ is the convolution in the orientation space, $w_{a b}^{o}\left(v_{b}\right)$ is a synaptic plasticity factor that modulates the effective synaptic strengths with the presynaptic activity, $p_{a E}$ and $p_{a I}$ are the connection probability functions between populations, and $\mu$ represents a stimulus of orientation $\theta_{0}$ and contrast $c$. In the limit $N \rightarrow \infty$ and requiring that $\mu_{a}$ in Eq. (9) is finite, we get that

$$
\begin{gathered}
J_{E E} q p_{E} *\left(w_{E E}^{o} v_{E}\right)-J_{E I}(1-q) p_{I} *\left(w_{E I}^{o} v_{I}\right)+\mu=0, \\
J_{I E} q p_{E} *\left(w_{I E}^{o} v_{E}\right)-J_{I I}(1-q) p_{I} *\left(w_{I I}^{o} v_{I}\right)+\mu=0,
\end{gathered}
$$

where $q=\frac{N_{E}}{N}$ is the fraction of excitatory neurons and we have made the assumption that $p_{E E}=p_{I E}=p_{E}$ and $p_{E I}=$ $p_{I I}=p_{I}$. The probability of connection is given by $p_{b}(\theta-$ $\psi)=\frac{\bar{p}_{b}}{\sigma_{b} \sqrt{2 \pi}} e^{-\frac{(\theta-\psi)^{2}}{2 \sigma_{b}^{2}}}$, where $\theta-\psi$ denotes difference in preferred orientation and $\sigma_{b}$ denotes connectivity width. We model the input $\mu$ as a Gaussian function,

$$
\mu=c e^{-\frac{\left(\theta-\theta_{0}\right)^{2}}{2 \sigma_{\mathrm{stim}}^{2}}},
$$

where $c$ is the stimulus contrast, $\sigma_{\text {stim }}$ is the tuning width of the input, and $\theta_{0}$ is the stimulus orientation (see Table $\mathrm{S} 1$ for parameters [29])

\section{Methods related to network models}

Model I describes homogeneously randomly connected networks with constant synaptic weights (Fig. S1 [29]). The connection probabilities $p_{E}$ and $p_{I}$ are uniform across the feature space $\Gamma$. The synaptic plasticity factors are constant and equal unity, $w_{a b}^{0, i j}=w_{a b}^{o}=1$. The external feed-forward input $F_{\mathrm{ffw}}$ is orientation independent, with $\mu=c$.

Model II describes homogeneously randomly connected networks with plastic $E \rightarrow E$ synaptic weights (Fig. 3). The synaptic plasticity factor $w_{E E}^{0, i j}$ models STP as in Refs. [25,48]. The firing rate approximation for $w_{E E}^{o}$ in STP is given in the Supplemental Material [29]. The synaptic plasticity factors for the remaining population connectivities are constant and equal unity. The connection probabilities $p_{E}$ and $p_{I}$ are constant. The external feed-forward input $F_{\mathrm{ffw}}$ is orientation independent, with $\mu=c$.

Model III describes networks of neurons connected with orientation-dependent probability and constant synaptic weights [Fig. S4 [29], Eq. (10)]. For each neuron, $C_{a}$ presynaptic neurons are sampled from a probability distribution $p_{a b}(\theta-\psi)$, which is described by a Gaussian function of width $\sigma_{a}^{2}$. Therefore the number of presynaptic inputs to a neuron for a particular network size $N$ is the same as in models I and II, but here the majority of connections are made within the nearby orientation space. The synaptic plasticity factors $w_{a b}^{0, i j}$ and $w_{a b}^{o}$ are constant and equal unity.

Model IV describes networks of neurons connected with orientation-dependent probability and plastic $E \rightarrow E$ synaptic weights [Fig. 4, Eq. (5)]. The connectivity is implemented as in model III, while the synaptic plasticity factors $w_{E E}^{0, i j}$ and $w_{a b}^{o}$ are defined as in model II.

Model $V$ describes networks of neurons connected with orientation-dependent probability (see model III) and with a power-law type of synaptic plasticity in the $E \rightarrow E$ and $E \rightarrow I$ connections [Fig. 5, Eq. (6)]:

$$
w_{E E}^{0, i j}=w_{I E}^{0, i j}=\left(\frac{v_{b, j}}{v_{0}}\right)^{\mathrm{k}},
$$


where $v_{0}=1 \mathrm{~Hz}$, and $\mathrm{k}$ denotes the synaptic efficacy. The firing rate $v_{b, j}$ of neuron $j$ is estimated using the sum of its last $n=10$ interspike intervals (ISIs) (see Supplemental Material for details [29]):

$$
v_{b, j}=\frac{n-1}{\sum_{s=1}^{n} I S I_{s}} .
$$

Model VI describes networks of neurons connected with orientation-dependent probability (see model III) and with STP in the $E \rightarrow E$ and $E \rightarrow I$ connections (Fig. 6, S6 [29]). The synaptic plasticity factors $w_{E E}^{0, i j}$ and $w_{I E}^{0, i j}$ are defined as in model II. The network in Fig. 6 has two excitatory subpopulations, one is connected to other excitatory and inhibitory neurons through facilitating STP synapses and the other through depressing STP synapses. The synaptic plasticity factors for the remaining population connectivities are constant and equal unity. [29].

Parameters for each network model are given in Table S1

\section{Mathematical details on the susceptibility to input contrast $\delta$ in uniform and randomly connected networks with $E \rightarrow E$ plasticity}

Here we consider $E \rightarrow E$ plasticity (see Supplemental Material for analogous calculations for networks with $E \rightarrow E$ and $E \rightarrow I$ plastic synapses [29]). From Eq. (10) we define the input contrast $c$ that is consistent with a balanced network with uniform random connectivity and $E \rightarrow E$ plastic synapses to fire at rate $v_{E}\left(w_{E I}^{o}=w_{I E}^{o}=w_{I I}^{o}=1\right)$ :

$$
c\left(v_{E}\right)=v_{E} q p_{E} \frac{J_{E I} J_{I E}-J_{I I} J_{E E} w_{E E}^{o}}{J_{I I}-J_{E I}} .
$$

Note that for uniform connectivity $p_{E}$ and $p_{I}$ and firing rates $v_{E}$ and $v_{I}$, the convolutions in Eq. (10) simplify to a product. Using the definition of $\mathrm{k}_{E E}$ [Eq. (4)], the derivative of the input contrast $c$ with respect to the excitatory firing rate $v_{E}$ is inserted into Eq. (1) to obtain

$$
\delta=1+\frac{w_{E E}^{o} k_{E E}}{\beta-w_{E E}^{o}\left(1+k_{E E}\right)},
$$

where $\beta=\frac{J_{E I} J_{I E}}{J_{I I} J_{E E}}$. This equation is equivalent to Eq. (2), where $w_{E E}=\frac{w_{E E}^{o}}{\beta}$. We introduce the normalized $E \rightarrow E$ synaptic factor $w_{E E}=\frac{w_{E E}^{o}}{\beta}$ to get

$$
\delta=\frac{1-w_{E E}}{1-w_{E E}\left(1+k_{E E}\right)} .
$$

The phase space of values for $\delta$ is shown in Fig. 2 .

\section{ACKNOWLEDGMENTS}

This work was funded by the Max Planck Society, University of Bonn Medical Center, University of Mainz Medical Center, and the German Research Foundation via CRC 1080 (T.T.). A.N. and L.BT. acknowledge additional support from an add-on scholarship of the Joachim Herz Foundation. We thank Nataliya Kraynyukova, Carlos Wert Carvajal, and Simon Renner for commenting on earlier versions of the manuscript, Alexander Dutine for validating the code, and members of the Tchumatchenko Laboratory for fruitful discussions. We acknowledge the support of the Center for Multiscale Modelling in Life Sciences (CMMS) by the LOEWE initiative of the Hessian Government. L.BT. and T.T. conceived the study. L.BT. and S.K. developed the theoretical framework. L.BT. performed the analytic calculations, the numerical simulations, and analyzed the data. P.E., A.N., and S.K. wrote the spiking network code. P.E., A.N., and L.BT. validated the code. L.BT. wrote the first draft of the manuscript. L.BT., A.N., P.E., and T.T wrote the manuscript. All authors provided critical feedback and helped shape the research and analysis.

The authors declare no competing interests.
[1] D. A. Leopold, Primary visual cortex: Awareness and blindsight, Annu. Rev. Neurosci. 35, 91 (2012).

[2] F. Tong, Primary visual cortex and visual awareness, Nat. Rev. Neurosci. 4, 219 (2003).

[3] A. F. Rossi, C. D. Rittenhouse, and M. A. Paradiso, The representation of brightness in primary visual cortex, Science 273, 1104 (1996).

[4] G. Sclar and R. D. Freeman, Orientation selectivity in the cat's striate cortex is invariant with stimulus contrast, Exp. Brain Res. 46, 457 (1982).

[5] C. E. Schreiner, Spatial distribution of responses to simple and complex sounds in the primary auditory cortex, Audiol. Neurotol. 3, 104 (1998).

[6] K. A. Bolding and K. M. Franks, Recurrent cortical circuits implement concentration-invariant odor coding, Science 361, 6407 (2018).
[7] J. S. Anderson, M. Carandini, and D. Ferster, Orientation tuning of input conductance, excitation, and inhibition in cat primary visual cortex, J. Neurophysiol. 84, 909 (2000).

[8] D. G. Laing, P. K. Legha, A. L. Jinks, and I. Hutchinson, Relationship between molecular structure, concentration, and odor qualities of oxygenated aliphatic molecules, Chem. Senses 28, 57 (2003).

[9] D. H. Hubel and T. N. Wiesel, Receptive fields, binocular interaction, and functional architecture in the cat's visual cortex, J. Physiol. 160, 106 (1962).

[10] D. Ferster and K. D. Miller, Neural mechanisms of orientation selectivity in the visual cortex, Annu. Rev. Neurosci. 23, 441 (2000).

[11] D. A. Wilson, Rapid, experience-induced enhancement in odorant discrimination by anterior piriform cortex neurons, J. Neurophysiol. 90, 65 (2003). 
[12] D. Moshitch, L. Las, N. Ulanovsky, O. Bar-Yosef, and I. Nelken, Responses of neurons in primary auditory cortex (A1) to pure tones in the halothane-anesthetized cat, J. Neurophysiol. 95, 3756 (2006).

[13] H. Ko, S. B. Hofer, B. Pichler, K. A. Buchanan, P. J. Sjöström, and T. D. Mrsic-Flogel, Functional specificity of local synaptic connections in neocortical networks, Nature (London) 473, 87 (2011).

[14] D. V. Buonomano and M. M. Merzenich, Cortical plasticity: From synapses to maps, Annu. Rev. Neurosci. 21, 149 (1998).

[15] J. A. Varela, K. Sen, J. Gibson, J. Fost, L. F. Abbott, and S. B. Nelson, A quantitative description of short-term plasticity at excitatory synapses in layer $2 / 3$ of rat primary visual cortex, J. Neurosci. 17, 7926 (1997).

[16] L. Busse, A. R. Wade, and M. Carandini, Representation of concurrent stimuli by population activity in visual cortex, Neuron 64, 931 (2009).

[17] M. Carandini and F. Sengpiel, Contrast invariance of functional maps in cat primary visual cortex, J. Vision 4, 1 (2004).

[18] P. Heil, R. Rajan, and D. R. F. Irvine, Topographic representation of tone intensity along the isofrequency axis of cat primary auditory cortex, Hearing Res. 76, 188 (1994).

[19] S. Uppenkamp and M. Röhl, Human auditory neuroimaging of intensity and loudness, Hearing Res. 307, 65 (2014).

[20] I. M. Finn, N. J. Priebe, and D. Ferster, The emergence of contrast-invariant orientation tuning in simple cells of cat visual cortex, Neuron 54, 137 (2007).

[21] C. van Vreeswijk and H. Sompolinsky, Chaos in neuronal networks with balanced excitatory and inhibitory activity, Science 274, 1724 (1996).

[22] R. Rosenbaum and B. Doiron, Balanced Networks of Spiking Neurons with Spatially Dependent Recurrent Connections, Phys. Rev. X 4, 021039 (2014).

[23] M. Okun and I. Lampl, Instantaneous correlation of excitation and inhibition during ongoing and sensory-evoked activities, Nat. Neurosci. 11, 535 (2008).

[24] D. Hansel and C. van Vreeswijk, The mechanism of orientation selectivity in primary visual cortex without a functional map, J. Neurosci. 32, 4049 (2012)

[25] G. Mongillo, D. Hansel, and C. van Vreeswijk, Bistability and Spatiotemporal Irregularity in Neuronal Networks with Nonlinear Synaptic Transmission, Phys. Rev. Lett. 108, 158101 (2012).

[26] The spiking network code was written in $\mathrm{C}++$ and the results analyzed in MATLAB (Matlab 2018b, Mathworks). The code is available at https://gitlab.rlp.net/braincodepublished/neuronalnetwork-simulations-June-2021.

[27] R. B. Levy and A. D. Reyes, Spatial profile of excitatory and inhibitory synaptic connectivity in mouse primary auditory cortex, J. Neurosci. 32, 5609 (2012).

[28] D. Hansel and G. Mato, Short-term plasticity explains irregular persistent activity in working memory tasks, J. Neurosci. 33, 133 (2013).

[29] See Supplemental Material at http://link.aps.org/supplemental/ 10.1103/PhysRevResearch.4.013162 for details on the shortterm plasticity model, the network susceptibility, and its firing rate, and the stability of the steady states. Supplemental figures referenced in the main text are also contained in the Supplemental Material.

[30] A. M. Thomson and J. Deuchars, Temporal and spatial properties of local circuits in neocortex, Trends Neurosci. 17, 119 (1994).

[31] W. R. Softky and C. Koch, The highly irregular firing of cortical cells is inconsistent with temporal integration of random EPSPs, J. Neurosci. 13, 334 (1993).

[32] D. Hansel and C. van Vreeswijk, How noise contributes to contrast invariance of orientation tuning in cat visual cortex, J. Neurosci. 22, 5118 (2002).

[33] K. D. Miller and T. W. Troyer, Neural noise can explain expansive, power-law nonlinearities in neural response functions, J. Neurophysiol. 87, 653 (2002).

[34] M. Carandini, Melting the iceberg: Contrast invariance in visual cortex, Neuron 54, 11 (2007).

[35] Y. Ahmadian and K. D. Miller, What is the dynamical regime of cerebral cortex? Neuron 109, 3373 (2021).

[36] Y. Ahmadian, D. B. Rubin, and K. D. Miller, Analysis of the stabilized supralinear network, Neural Comput. 25, 1994 (2013).

[37] D. B. Rubin, S. D. Van Hooser, and K. D. Miller, The stabilized supralinear network: A unifying circuit motif underlying multi-input integration in sensory cortex, Neuron 85, 402 (2015).

[38] A. Sanzeni, M. H. Histed, and N. Brunel, Response nonlinearities in networks of spiking neurons, PLoS Comput. Biol. 16, e1008165 (2020).

[39] G. H. Recanzone, D. C. Guard, and M. L. Phan, Frequency, and intensity response properties of single neurons in the auditory cortex of the behaving macaque monkey, J. Neurophysiol. 83, 2315 (2000).

[40] C. E. Schreiner, H. L. Read, and M. L. Sutter, Modular organization of frequency integration in primary auditory cortex, Annu. Rev. Neurosci. 23, 501 (2000).

[41] J. F. Linden, R. C. Liu, M. Sahani, C. E. Schreiner, and M. M. Merzenich, Spectrotemporal structure of receptive fields in areas AI and AAF of mouse auditory cortex, J. Neurophysiol. 90, 2660 (2003).

[42] A. Y. Y. Tan, L. I. Zhang, M. M. Merzenich, and C. E. Schreiner, Tone-evoked excitatory and inhibitory synaptic conductances of primary auditory cortex neurons, J. Neurophysiol. 92, 630 (2004).

[43] T. Branco and K. Staras, The probability of neurotransmitter release: Variability and feedback control at single synapses, Nat. Rev. Neurosci. 10, 373 (2009).

[44] N. J. Priebe and D. Ferster, Inhibition, spike threshold, and stimulus selectivity in primary visual cortex, Neuron 57, 482 (2008).

[45] M. Carandini, Amplification of trial-to-trial response variability by neurons in visual cortex, PLoS Biology 2, e264 (2004).

[46] R. J. Douglas and K. A. C. Martin, Neuronal circuits of the neocortex, Annu. Rev. Neurosci. 27, 419 (2004).

[47] A. J. Rockel, R. W. Hiorns, and T. P. Powell, The basic uniformity in structure of the neocortex, Brain: J. Neurol. 103, 221 (1980).

[48] M. V. Tsodyks and H. Markram, The neural code between neocortical pyramidal neurons depends on neurotransmitter release probability, Proc. Natl. Acad. Sci. U.S.A. 94, 719 (1997). 
[49] L. F. Abbott, J. A. Varela, K. Sen, and S. B. Nelson, Synaptic depression and cortical gain control, Science 275, 221 (1997).

[50] J. G. G. Borst, The low synaptic release probability in vivo, Trends Neurosci. 33, 259 (2010).

[51] A. Pala and C. C. H. Petersen, In vivo measurement of cell-typespecific synaptic connectivity and synaptic transmission in layer 2/3 mouse barrel cortex, Neuron 85, 68 (2015).

[52] J.-S. Jouhanneau, J. Kremkow, A. L. Dorrn, and J. F. A. Poulet, In vivo monosynaptic excitatory transmission between layer 2 cortical pyramidal neurons, Cell Reports 13, 2098 (2015).

[53] J.-S. Jouhanneau and J. F. A. Poulet, Multiple two-photon targeted whole-cell patch-clamp recordings from monosynaptically connected neurons in vivo, Front. Synaptic Neurosci. 11, 15 (2019).

[54] E. Marder, Neuromodulation of neuronal circuits: Back to the future, Neuron 76, 1 (2012).
[55] R. Metherate, N. Tremblay, and R. W. Dykes, The effects of acetylcholine on response properties of cat somatosensory cortical neurons, J. Neurophysiol. 59, 1231 (1988).

[56] T. M. McKenna, J. H. Ashe, and N. M. Weinberger, Cholinergic modulation of frequency receptive fields in auditory cortex: I. Frequency-specific effects of muscarinic agonists, Synapse 4, 30 (1989).

[57] M. I. Kerr, M. J. Wall, and M. J. E. Richardson, Adenosine a1 receptor activation mediates the developmental shift at layer 5 pyramidal cell synapses and is a determinant of mature synaptic strength, J. Physiol. 591, 3371 (2013).

[58] G. Qi, K. van Aerde, T. Abel, and D. Feldmeyer, Adenosine differentially modulates synaptic transmission of excitatory and inhibitory microcircuits in layer 4 of rat barrel cortex, Cerebral Cortex 27, 4411 (2017).

[59] D. L. Meinecke and A. Peters, GABA immunoreactive neurons in rat visual cortex, J. Comp. Neurol. 261, 388 (1987). 